\title{
Synchronization boost with single-photon dissipation in the deep quantum regime
}

\author{
W.-K. Mok, ${ }^{1}$ L.-C. Kwek, ${ }^{1,2,3,4}$ and H. Heimonen ${ }^{1}$ \\ ${ }^{1}$ Centre for Quantum Technologies, National University of Singapore, 3 Science Drive 2, Singapore 117543 \\ ${ }^{2}$ MajuLab, CNRS-UNS-NUS-NTU International Joint Research Unit, UMI 3654, Singapore \\ ${ }^{3}$ National Institute of Education, Nanyang Technological University, 1 Nanyang Walk, Singapore 637616 \\ ${ }^{4}$ School of Electrical and Electronic Engineering, Block S2.1, 50 Nanyang Avenue, Singapore 639798
}

(Received 20 February 2020; accepted 21 August 2020; published 16 September 2020)

\begin{abstract}
Synchronization phenomena occur throughout nature. The van der Pol oscillator has been a paradigmatic model to investigate synchronization. Here we study the oscillator with additional single-photon dissipation in the deep quantum regime (defined to be $\gamma_{2} / \gamma_{1} \gtrsim 10$ ), and we contrast it with the quantum regime at $\gamma_{2} / \gamma_{1} \approx 1$. Our results show that in this regime: (i) the effect of squeezed driving effect on frequency entrainment is strongly suppressed, (ii) single-photon dissipation boosts synchronization, (iii) synchronization is bounded, and (iv) the limit-cycle is robust and insensitive to strong driving. We use these physical properties to define the crossover to the deep quantum regime. We also propose a synchronization measure based on directional statistics which is analytically calculated. These results reflect the intrinsic physical differences between synchronization in the quantum and deep quantum regimes.
\end{abstract}

DOI: 10.1103/PhysRevResearch.2.033422

\section{INTRODUCTION}

From coupled metronomes [1-3] to brain activity [4] and jet-lag [5], synchronization is used to explain countless phenomena in nature. In practice, many of these processes are noisy, with random noise influencing the dynamical systems. This has given rise to many noise-enhanced, and noise-enabled processes, such as signal amplification [6] and stochastic resonance $[7,8]$. Studies of synchronization have been taken to the quantum domain and various quantum systems with limit cycles have been studied in recent years, including the weakly nonlinear quantum van der Pol (qvdP) oscillator [9-16], optomechanical systems [17-22], and lowdimensional systems [23-28]. Similar phenomena have also been investigated with superconducting circuits $[29,30]$. Interest lies in understanding the fundamental differences between quantum and classical synchronization [12,13,31], realizing a quantum self-sustained oscillator [25,32], and using quantum synchronization for tasks such as operating heat engines [33], and generating entanglement $[34,35]$.

In this paper we push the qvdP into the deep quantum regime (characterised by $\gamma_{2} / \gamma_{1} \gtrsim 10$ ), and the deep quantum limit $\left(\gamma_{2} / \gamma_{1} \rightarrow \infty\right)$. The classification of the quantum regime, deep quantum regime, and (deep) quantum limit have so far been inconsistent and unclear referring to values of $\gamma_{2} / \gamma_{1}$ from 1 to 1000 and $\infty$ [9-11,13,14,20,24,31,34,36-40]. Here we define the quantum and deep quantum regimes based on

Published by the American Physical Society under the terms of the Creative Commons Attribution 4.0 International license. Further distribution of this work must maintain attribution to the author(s) and the published article's title, journal citation, and DOI. the different physics of the qvdP for values of $\gamma_{2} / \gamma_{1}$ on either side of approximately 10 . We propose to separate the various regimes of quantum synchronization into several sectors on physical grounds, as summarised in Table I. The different features of synchronization in the (deep) quantum regime will be discussed later in the paper.

Experimentally, single-photon losses cannot be removed from the oscillator. Yet, we find that it can boost synchronization in the deep quantum regime, and we show that such a boost cannot be observed with single-photon dissipation in the semiclassical and classical regimes. We also find that the amount of synchronization to an external drive is bounded in the deep quantum regime, even for arbitrary driving strength. Moreover, we discover that the effect of a squeezing drive is strongly suppressed in this regime and we obtain an upper bound to the amount of synchronization for a harmonically driven qvdP. The qvdP limit cycle is also shown to be much more robust against perturbation from driving compared with its classical counterpart.

\section{MODEL}

To study quantum synchronization, we require a selfsustained (limit-cycle) oscillator. Synchronous behavior of a quantum system without a limit cycle does not necessarily count as a quantum analog of the well-known classical synchronization, since the phase of the system is not free [3]. To this end, the most commonly studied model is the quantum van der Pol oscillator in the weakly nonlinear regime $[9,10]$, also known as the quantum Stuart-Landau oscillator [41]. We add linear damping (i.e., single-photon dissipation) to the standard qvdP, fully described by the master equation

$$
\dot{\rho}=-i[H, \rho]+\gamma_{1} \mathcal{D}\left[a^{\dagger}\right] \rho+\gamma_{2} \mathcal{D}\left[a^{2}\right] \rho+\kappa \mathcal{D}[a] \rho,
$$


TABLE I. Classifying the different regimes of the qvdP, based on the physical phenomena arising as we move down the table. Different analytical methods are also needed to describe the oscillator in the different regimes. Note that the list of features in the final column is not exhaustive.

\begin{tabular}{|c|c|c|c|}
\hline Regime & $\gamma_{2} / \gamma_{1}$ & Analytical Method & Selected Features \\
\hline Classical limit & 0 & Mean-field [1-3] & Noiseless oscillator [1-3] \\
\hline Semi-classical regime & $\lesssim 0.1$ & $\begin{array}{l}\text { System size expansion }[9,45,46] \\
\text { Phase reduction theory }[47]\end{array}$ & Gaussian noise [45] \\
\hline Quantum regime & 1 & None & $\begin{array}{l}\text { Synchronization blockade [12] } \\
\text { Squeezing enhancement [31] }\end{array}$ \\
\hline Deep quantum regime & $\gtrsim 10$ & $\begin{array}{l}\text { Density matrix ansatz (this work) } \\
\text { Perturbation theory [24] }\end{array}$ & $\begin{array}{l}\text { Dissipative synchronization boost } \\
\text { (this work) } \\
\text { Bounded synchronization } \\
\text { (this work, [24]) } \\
\text { Suppression of squeezing enhancement } \\
\text { (this work, [24]) }\end{array}$ \\
\hline Deep quantum limit & $\infty$ & $\begin{array}{l}\text { Density matrix ansatz (this work) } \\
\text { Density matrix approach in a } \\
\text { truncated Hilbert space of a qubit [38] } \\
\text { Perturbative solution to Wigner } \\
\text { function equation of motion [9] }\end{array}$ & $\begin{array}{l}\text { Synchronization determined by } \\
\text { single coherence }[14,38]\end{array}$ \\
\hline
\end{tabular}

with the Hamiltonian given by

$$
H=\delta a^{\dagger} a+\Omega\left(a+a^{\dagger}\right)+\eta\left(a^{2}+a^{\dagger 2}\right)
$$

in the rotating frame with the harmonic and squeezing drives, using the rotating-wave approximation. The Lindblad superoperator in the master equation is defined by $\mathcal{D}[\hat{O}] \rho \equiv$ $O \rho O^{\dagger}-\frac{1}{2}\left\{O^{\dagger} O, \rho\right\}$. The qvdP is driven by a harmonic drive of strength $\Omega$ and a squeezing Hamiltonian of strength $\eta$. The oscillator detuning is $\delta=\omega_{0}-\omega_{d}$, where $\omega_{0}$ is the natural frequency of the oscillator and $\omega_{d}$ is the driving frequency. The limit cycle is maintained by three incoherent processes: single-photon pumping with the pumping rate $\gamma_{1}$, two-photon loss with decay rate $\gamma_{2}$, and an additional single-photon loss with decay rate $\kappa$. These incoherent processes do not imprint any phase preference on the oscillator, leaving the phase free and enabling the oscillator to synchronize.

\section{ANALYTICS}

The optical phase is a periodic variable in the interval $[0,2 \pi)$. Therefore, it is natural to use directional statistics [42] to study its properties. To measure phase synchronization we use the mean resultant length (MRL) of a circular distribution $S=\sqrt{\langle\sin \phi\rangle^{2}+\langle\cos \phi\rangle^{2}}=\left|\left\langle e^{i \phi}\right\rangle\right|$, where the expectation value is taken over the probability distribution. The MRL, closely related to the circular variance [42], is used to measure synchronization for classical noisy systems [43], and is also famously the order parameter of the Kuramoto model [41]. We choose the MRL as our synchronization measure for its suitable properties: (i) It takes the value 0 for an unsynchronized state, and the value 1 for a perfectly synchronized state where the phase distribution is a $\delta$ function. Thus, the MRL has a clear numerical meaning, unlike unbounded measures. Such measures, like the peak of the phase distribution [12], that go to infinity in the (classical) noiseless case are harder to interpret and compare across systems. (ii) The MRL has a natural counterpart as the expectation value of a quantum operator, given by $S=\left|\left\langle e^{i \hat{\phi}}\right\rangle\right|=\left|\operatorname{Tr}\left(e^{i \hat{\phi}} \rho\right)\right|$, where $\hat{\phi}$ is the phase operator defined by Pegg and Barnett [44]. (iii) Having a quantum analog allows for analytical calculations of the amount of synchronization in the system. Another feature of synchronization is frequency entrainment. It is quantified by the shifting of the peak of the frequency spectrum.

With pure harmonic driving of the qvdP $(\eta=0)$, we can obtain an analytical approximation for the master equation in the deep quantum regime. To this end, we make the following ansatz for the density matrix in the Fock basis:

$$
\rho_{s s}=\left(\begin{array}{ccc}
\rho_{00} & \rho_{01} & 0 \\
\rho_{10} & \rho_{11} & 0 \\
0 & 0 & \rho_{22}
\end{array}\right)
$$

This amounts to restricting the number of excitations to 2, and neglecting all coherences involving the state $|2\rangle$. The higher order coherences are dropped on the grounds that they can be seen to be small in exact numerical simulations, and dropping them makes analytical calculations much easier and more insightful. The accuracy of this approximation will be benchmarked later. The limit-cycle amplitude $N=\left\langle a^{\dagger} a\right\rangle=$ $\rho_{11}+2 \rho_{22}$ can now easily be calculated:

$$
\lim _{\gamma_{2} / \gamma_{1} \rightarrow \infty} N=\frac{\gamma_{1}\left(6 \gamma_{1} \kappa+9 \gamma_{1}^{2}+4 \delta^{2}+\kappa^{2}+12 \Omega^{2}\right)+4 \kappa \Omega^{2}}{\left(3 \gamma_{1}+\kappa\right)\left(6 \gamma_{1} \kappa+9 \gamma_{1}^{2}+4 \delta^{2}+\kappa^{2}+8 \Omega^{2}\right)},
$$

where the undriven amplitude is given by

$$
N_{0}=\frac{\gamma_{1}\left(2 \gamma_{1}+\gamma_{2}+\kappa\right)}{\gamma_{1}\left(3 \gamma_{2}+\kappa\right)+\kappa\left(\gamma_{2}+\kappa\right)+\gamma_{1}^{2}}
$$




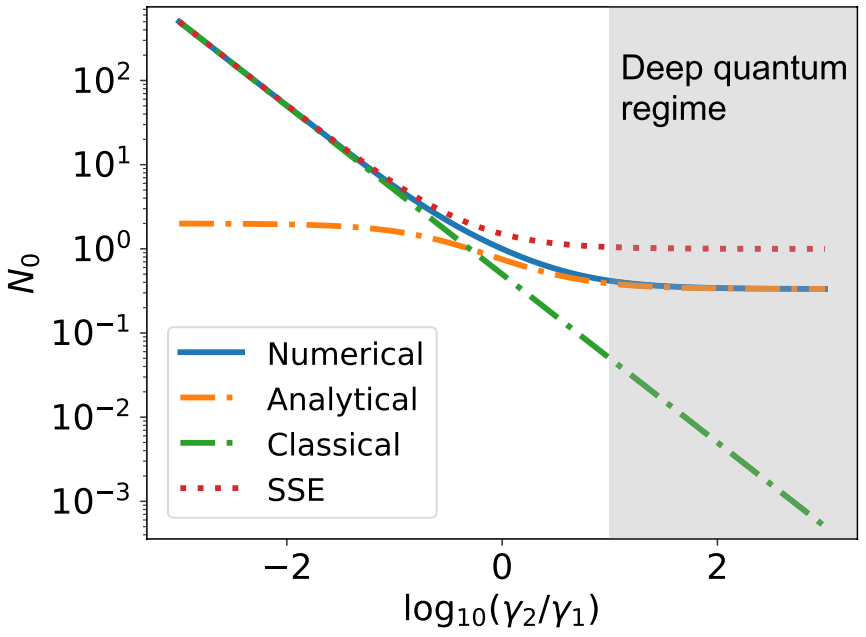

FIG. 1. Amplitude $N_{0}$ of the undriven standard qvdP oscillator. Analytical solution given by Eq. (5) (orange dash-dotted line) agrees well with the numerical simulations in the deep quantum regime $\gamma_{2} / \gamma_{1} \gtrsim 10$ (blue solid line). The classical curve refers to a mean-field calculation, and SSE refers to a semiclassical system-size expansion.

which simplifies in the deep quantum limit to

$$
\lim _{\gamma_{2} / \gamma_{1} \rightarrow \infty} N_{0}=\frac{\gamma_{1}}{3 \gamma_{1}+\kappa}
$$

As a check for consistency, we note that setting $\kappa=0$ in Eq. (6) gives $N_{0}=1 / 3$, which agrees with the well known result for the undriven qvdP in the deep quantum limit: $\lim _{\gamma_{2} / \gamma_{1} \rightarrow \infty} \rho_{s s}=\frac{2}{3}|0\rangle\left\langle 0\left|+\frac{1}{3}\right| 1\right\rangle\langle 1|[9,14]$.

In Fig. 1 we compare the standard $(\kappa=0)$ oscillator amplitude $N_{0}$ calculated numerically for various $\gamma_{2} / \gamma_{1}$ with three analytical approximations. The classical calculation refers to a mean-field approximation $\langle\hat{a}\rangle=\alpha$ that discards all quantum fluctuations. This approximation works well in the large amplitude limit, but breaks down as $\gamma_{2} / \gamma_{1} \approx 1$. A semiclassical calculation using a system size expansion (SSE) of the equation of motion of the Wigner function $[45,46]$ produces the expression $N_{0}=\left(\gamma_{1}+2 \gamma_{2}-\kappa\right) /\left(2 \gamma_{2}\right)$. The SSE performs better than the classical result of $N_{0}=\left(\gamma_{1}-\kappa\right) /\left(2 \gamma_{2}\right)$ in the quantum regime, but it too fails in the deep quantum regime as its limiting value is $N_{0}=1$, not $N_{0}=1 / 3$. Finally, the calculation in the deep quantum limit in Eq. (5) works well for $\gamma_{2} / \gamma_{1} \gtrsim 10$. This is the first reason for defining the deep quantum regime to start at approximately the damping ratio 10 . The synchronization measure is then identified from Eq. (3) as simply the coherence $\left|\rho_{01}\right|$, which can be found by solving for the steady-state density matrix elements, which are given in Appendix A. In the standard case with no single-photon dissipation $(\kappa=0)$, the synchronization measure is given by

$$
\lim _{\kappa / \gamma_{1} \rightarrow 0} S=\frac{2 \gamma_{2} \Omega \sqrt{9 \gamma_{1}^{2}+4 \delta^{2}}}{4 \gamma_{1}\left(\delta^{2}+3 \Omega^{2}\right)+3 \gamma_{2}\left(9 \gamma_{1}^{2}+4 \delta^{2}+8 \Omega^{2}\right)+9 \gamma_{1}^{3}} .
$$
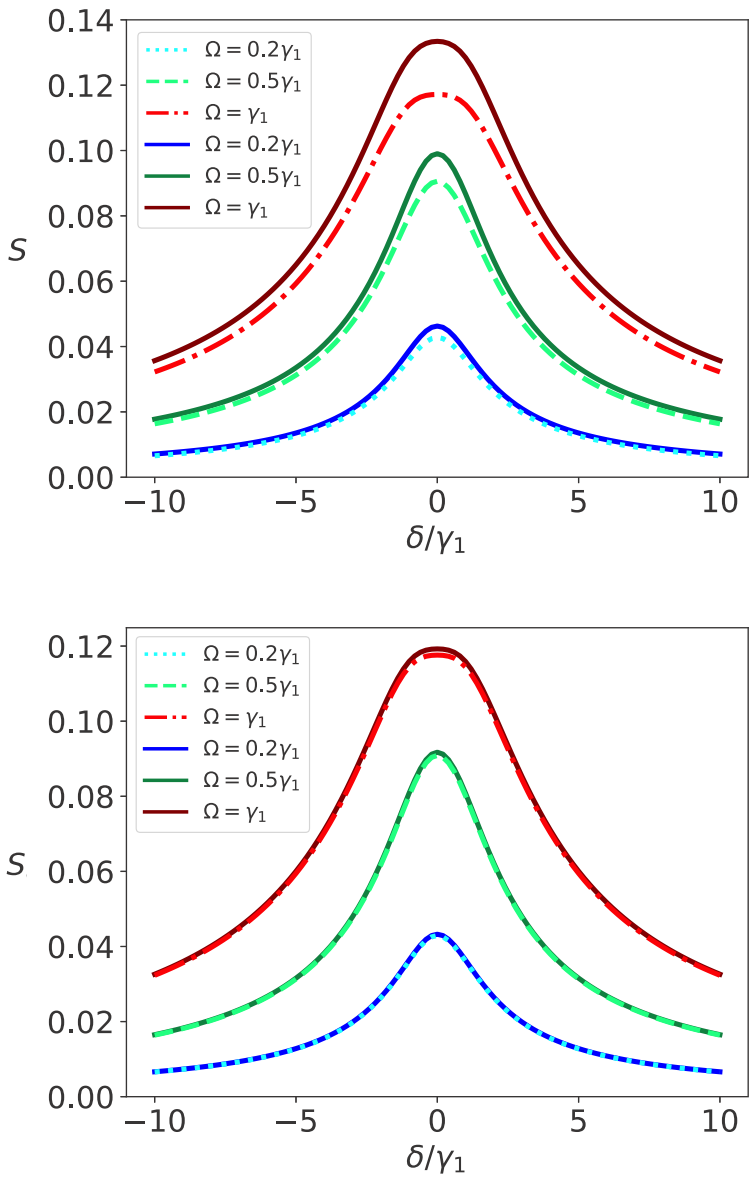

FIG. 2. Slices of the Arnold tongue for $\gamma_{2} / \gamma_{1}=100$ (top) and $\gamma_{2} / \gamma_{1}=1000$ (bottom). The synchronization measure $S$ is plotted as a function of detuning $\delta$ (normalized by the single-photon loss rate $\gamma_{1}$ ) for various driving strengths $\Omega / \gamma_{1}$. The solid lines denote numerical results and the dotted, dashed and dash-dotted lines represent analytical predictions for $S$ using the Eq. (7). (Light blue, dark blue): $\Omega=0.2 \gamma_{1}$, (Light green, dark green): $\Omega=0.5 \gamma_{1}$, (Red, brown): $\Omega=\gamma_{1}$.

Correspondingly, in the deep quantum limit where $\gamma_{2} / \gamma_{1} \rightarrow$ $\infty$, we have

$$
\lim _{\gamma_{2} / \gamma_{1} \rightarrow \infty} S=\frac{2 \Omega\left(\gamma_{1}+\kappa\right) \sqrt{\left(3 \gamma_{1}+\kappa\right)^{2}+4 \delta^{2}}}{\left(3 \gamma_{1}+\kappa\right)\left(6 \gamma_{1} \kappa+9 \gamma_{1}^{2}+4 \delta^{2}+\kappa^{2}+8 \Omega^{2}\right)} .
$$

The Arnold tongue illustrates the synchronization region in parameter space by plotting the synchronization measure against the driving strength $\Omega / \gamma_{1}$ and detuning $\delta / \gamma_{1}$. Figure 2 compares the expression in Eq. (8) to numerical results for different damping ratios $\gamma_{2} / \gamma_{1}$, by plotting slices of the Arnold tongue for fixed $\Omega / \gamma_{1}$, thereby showing the accuracy of Eq. (8) in the deep quantum regime. The analytical approximation works relatively well and shows a more significant deviation from the numerical results when $\gamma_{2} / \gamma_{1}=100$ and $\Omega=\gamma_{1}$. This is due to the higher-order coherences induced by the driving being nonnegligible, breaking the assumption of the density matrix ansatz (more details in Appendix C). The approximation becomes more accurate for $\gamma_{2} / \gamma_{1}=1000$ under similar driving conditions. We can also obtain the phase 
distribution $P(\phi)$ by projecting the qvdP state onto the phase eigenstates: $|\phi\rangle=\sum_{n} e^{i n \phi}|n\rangle$

$$
P(\phi)=\frac{1}{2 \pi}\langle\phi|\rho| \phi\rangle=\frac{1}{2 \pi} \sum_{m, n=0}^{\infty} e^{i(n-m) \phi} \rho_{m n} .
$$

In the deep quantum limit, we can obtain a simple expression for the phase distribution

$$
\begin{aligned}
\lim _{\gamma_{2} / \gamma_{1} \rightarrow \infty} P(\phi)= & \frac{1}{2 \pi}\left[1-\frac{4 \Omega\left(\kappa+\gamma_{1}\right)}{6 \gamma_{1} \kappa+9 \gamma_{1}^{2}+4 \delta^{2}+\kappa^{2}+8 \Omega^{2}}\right. \\
& \left.\times \sqrt{1+\frac{4 \delta^{2}}{\left(\kappa+3 \gamma_{1}\right)^{2}}} \cos (\phi-\mu)\right] \\
\mu= & -\arctan \left(\frac{\kappa+3 \gamma_{1}}{2 \delta}\right) .
\end{aligned}
$$

It is a cardioid distribution [42] $P(x)=\frac{1}{2 \pi}[1-2 S \cos (x-$ $\mu)$ ], with MRL $=S$, and mean direction $\mu$. It is easy to see that the MRL of the distribution coincides with the synchronization measure in Eq. (8) calculated directly from the density matrix elements. This shows that the MRL, as statistically defined, can indeed be represented by the expectation value of a quantum operator. That enables easy numerical and analytical calculations of the amount of synchronization without projecting onto phase eigenstates and shows how synchronization is tied to the coherences of the density matrix. In this case, the expression for $S$ gives an upper bound to synchronization by a harmonic drive in the deep quantum regime at $\lim _{\{\kappa, \Omega\} / \gamma_{1} \rightarrow \infty} S=1 /(2 \sqrt{2}) \approx 0.35$ [noting that the synchronization peaks lie along the line $\Omega / \gamma_{1}=\left(\kappa / \gamma_{1}+\right.$ $3) / 2 \sqrt{2}$ ]. This is unlike the behavior of the (semi)classical vdP oscillator [47], which approaches perfect synchronization $(S=1)$ for arbitrarily strong driving. The values that can be attained for moderate driving and single-photon loss are shown in Fig. 3, with $\gamma_{2} / \gamma_{1}$ chosen at 1 and 100 to represent the quantum and deep quantum regimes respectively. The result also differs from that in Ref. [24] in that it is independent of deformation of the limit cycle, as will be shown later. The mean direction $\mu$, however, indicates the position of the phase distribution peak, and represents the phase difference between the qvdP and the drive. As $\mu$ is given by an arctangent function, it always has a solution. For $\delta=0$ the phase difference between the oscillator and drive will be 0 , and for other detunings they will pick up a phasedifference in $[-\pi / 2, \pi / 2]$. Interestingly, the phase difference in the deep quantum regime is independent of the driving strength. This stands out from the classical [2] and semiclassical regime [47], where the synchronization phase depends on the driving strength, but is alike spin systems where a similar independence has been discovered [24]. Classically, by driving harder, the phase difference eventually vanishes, whereas the quantum phase difference stays constant. The classical phase-difference also only has a solution in a range of detunings determined by the driving strength, whereas in the deep quantum regime it has a solution for arbitrary detuning. The expressions therefore set the qvdP in the deep quantum regime apart from classical oscillators, showing the robustness of quantum synchronization.
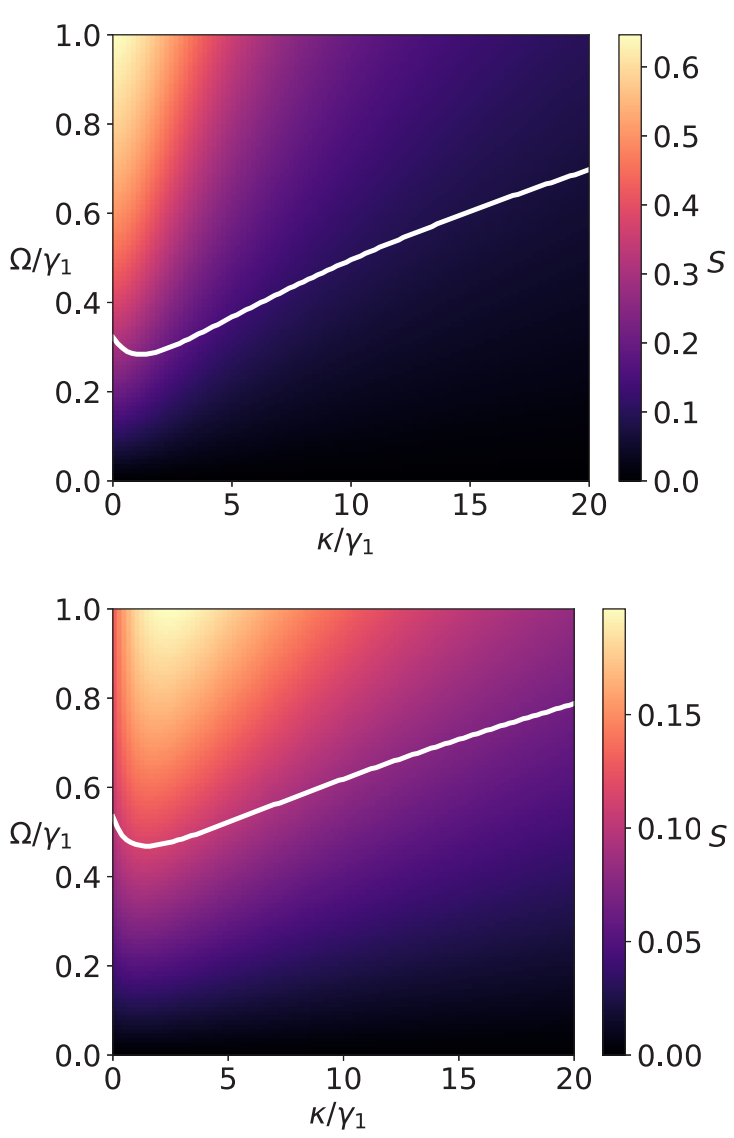

FIG. 3. Synchronization as a function of driving strength $\Omega$ and decay rate $\kappa$, calculated from numerical simulations of the master equation with $\delta=\eta=0$. The threshold driving $(\epsilon=0.1)$ is indicated by the white solid line. (Top) $\gamma_{2} / \gamma_{1}=1$, (Bottom) $\gamma_{2} / \gamma_{1}=$ 100 .

\section{SINGLE-PHOTON DISSIPATION BOOSTS SYNCHRONIZATION}

In a physical implementation of a qvdP, some excitation relaxation of the oscillator (for example, as spontaneous emission of phonons in mechanical oscillators or photons in optical cavities) is inevitable. This motivates considering nonnegligible relaxation rates $\kappa \neq 0$. Adding linear damping in the deep quantum regime can, counterintuitively, result in a boost in synchronization.

However, simply adding a nonzero $\kappa$ leads to a smaller limit cycle amplitude. To fairly compare synchronization with and without single-photon dissipation, we must keep the relative driving strengths constant. This is achieved by always setting the driving strength such that it distorts the limit cycle by a constant amount $\epsilon$, such that $\left|\Delta N / N_{0}\right|=\epsilon$, where $\Delta N \equiv N-N_{0}$. We can then compare the synchronization measures of the two oscillators. In the deep quantum regime, the threshold driving strength is found using Eq. (4):

$$
\Omega_{\mathrm{th}}^{2}=\frac{\epsilon\left(6 \gamma_{1} \kappa+9 \gamma_{1}^{2}+4 \delta^{2}+\kappa^{2}\right)}{4\left(\kappa+\gamma_{1}-2 \epsilon \gamma_{1}\right)} .
$$

Setting $\Omega / \gamma_{1}=1$ with resonant driving and varying $\kappa / \gamma_{1}$, we show numerically in Fig. 4 that the effect of single-photon loss differs dramatically in different regimes. The dissipation 

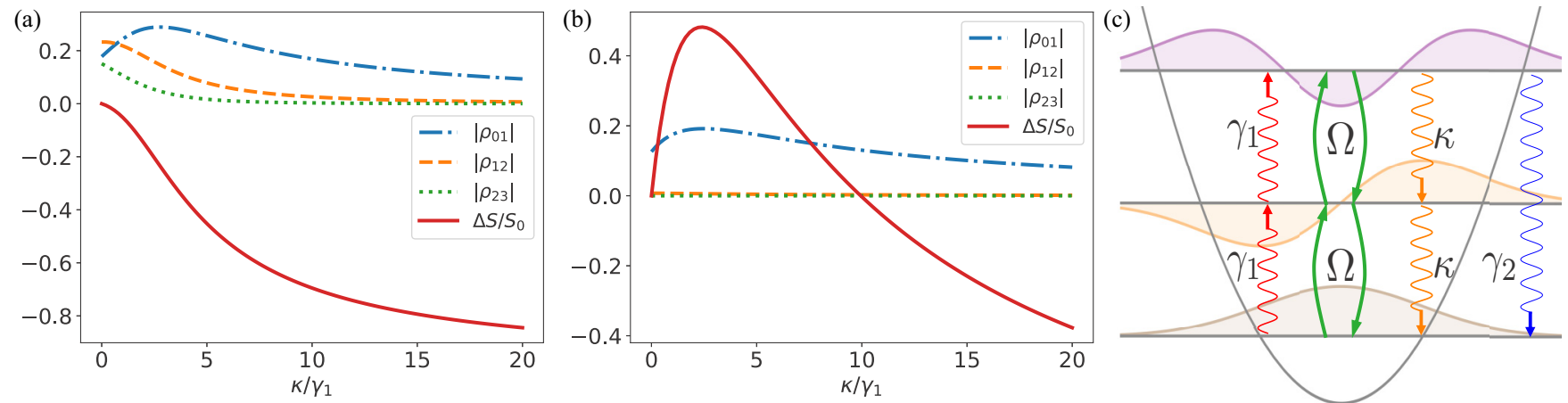

FIG. 4. Effects of single-photon loss on coherences and synchronization for (a) $\gamma_{2} / \gamma_{1}=1$ and (b) $\gamma_{2} / \gamma_{1}=100$. (c) Level diagram of the qvdP in the deep quantum regime. $\gamma_{1}$ shows the effect of the single-photon pumping, $\gamma_{2}$ the two-photon loss, $\kappa$ the single-photon loss, and $\Omega$ the coherent driving. In all cases, $\delta=0$ and $\Omega / \gamma_{1}=1$.

term $\kappa \mathcal{D}[a] \rho$ generally reduces coherences in the density matrix. The decoherence rate grows with the photon number, so higher elements in the density matrix decay faster, as seen in Figs. 4(a) and 4(b). In the deep quantum limit the synchronization is given by only the lowest coherence $\left|\rho_{01}\right|$, which increases with the addition of moderate amount of single-photon dissipation.

In Fig. 4(c) we see that the coherences are all built by the driving $\Omega$. The element $\left|\rho_{01}\right|$ then depends on the probability of the oscillator being in the state $|0\rangle$. In the absence of single-photon loss, once the oscillator has been driven into $|1\rangle$, it then has to be driven to the state $|2\rangle$ and decay via the two-photon loss before the driving can build up the coherence between the states $|0\rangle$ and $|1\rangle$. With weak driving, this is a very slow process. By adding the single-photon dissipation term, the oscillator can now decay down to the ground state $|0\rangle$ straight from $|1\rangle$. This increases the ground state population, which in turn increases the transition rate between $|0\rangle$ and $|1\rangle$. In the steady state, this shows up as a larger coherence $\left|\rho_{01}\right|$. Consequently, in the deep quantum regime, single-photon dissipation boosts synchronization significantly [ $>40 \%$ enhancement as shown in Fig. 4(b)].

Another way to understand the synchronization boost in the deep quantum regime is to rewrite the synchronization measure $S=\left|\rho_{01}\right|$ as

$$
S=\frac{2 \Omega}{\sqrt{4 \delta^{2}+\left(\kappa+3 \gamma_{1}\right)^{2}}} \times\left|\rho_{00}-\rho_{11}\right|
$$

(using the expressions for the density matrix elements in Appendix A), from which we observe that the amount of synchronization is directly proportional to the population imbalance between $|0\rangle$ and $|1\rangle$. In principle, by adding more single-photon pumping $\mathcal{D}\left[a^{\dagger}\right] \rho$, some of the qvdP population can be redistributed from $|0\rangle$ to $|1\rangle$, which would lead to greater synchronization. This corresponds to decreasing the $\gamma_{2} / \gamma_{1}$ ratio which decreases the "quantumness" of the qvdP. Hence, it is unsurprising that doing so will increase the amount of synchronization. However, what we are considering in this work is the synchronization boost for a fixed $\gamma_{2} / \gamma_{1}$. Naturally, by adding some single-photon loss $\mathcal{D}[a] \rho$, we can also increase the population imbalance by redistributing some population from $|1\rangle$ to $|0\rangle$, which explains the observed increase in synchronization. Note, however, that the presence of $\kappa$ in the denominator of Eq. (12) implies that $S$ will tend to zero as $\kappa \rightarrow \infty$ (with all the other parameters fixed), since $\left|\rho_{00}-\rho_{11}\right|$ is upper bounded by unity. This explains why there is an optimal value of $\kappa$ for the synchronization boost in Fig. 4(b).

In other words, adding single-photon dissipation gives rise to a competition between two processes: (i) the increase in lowest-order coherence $\left|\rho_{01}\right|$ due to the increased interaction between states $|0\rangle$ and $|1\rangle$ via the driving field, and (ii) decoherence which is more significant for higher-order coherences. Thus, when $\rho_{01}$ is the dominant coherence [particularly true in the deep quantum regime, see Fig. 4(b)], the first effect (i) can outweigh the decoherence (ii) for moderate dissipation rates $\kappa$, leading to a synchronization boost. The competition results in an optimal single-photon dissipation rate to boost synchronization. In the limit of large single-photon dissipation rate, the qvdP is essentially suppressed at the ground state $|0\rangle$ and is unable to couple to $|1\rangle$ via the driving field effectively, thus $\left|\rho_{01}\right|$ decreases. This explains why the synchronization is eventually killed off by the single-photon loss for large decay rate $\kappa \gg\left\{\Omega, \gamma_{1}\right\}$. However, if $\left|\rho_{01}\right|$ is not the dominant coherence (such as in Fig. 4(a)), i.e., in the quantum or (semi)classical regimes, then no synchronization boost occurs since the decay of all the higher coherence terms dominates over any increase in $\left|\rho_{01}\right|$ with $\kappa$.

In the deep quantum limit, the statement can be formalized by studying the behavior of Eq. (8) while varying $\kappa$ near $\kappa=0$ :

$$
\left.\frac{\partial S}{\partial \kappa}\right|_{\kappa=0}=\frac{2 \Omega\left(3 \gamma_{1}^{2}+8 \Omega^{2}\right)}{\left(9 \gamma_{1}^{2}+8 \Omega^{2}\right)^{2}}>0,
$$

for any fixed $\Omega>0$, there will be an initial synchronization boost when increasing $\kappa$ from zero. Note that this effect occurs purely due to the single-photon dissipation, and not due to the changing driving strength introduced in Eq. (11). This effect is not limited to only the deep quantum limit, but also possible in the deep quantum regime as well. We can also impose the condition $\partial_{\kappa} S(\kappa=0)>0$ on the full expression of the density matrix (more details provided in Appendix B) to find a necessary condition to observe the dissipation boost. The 


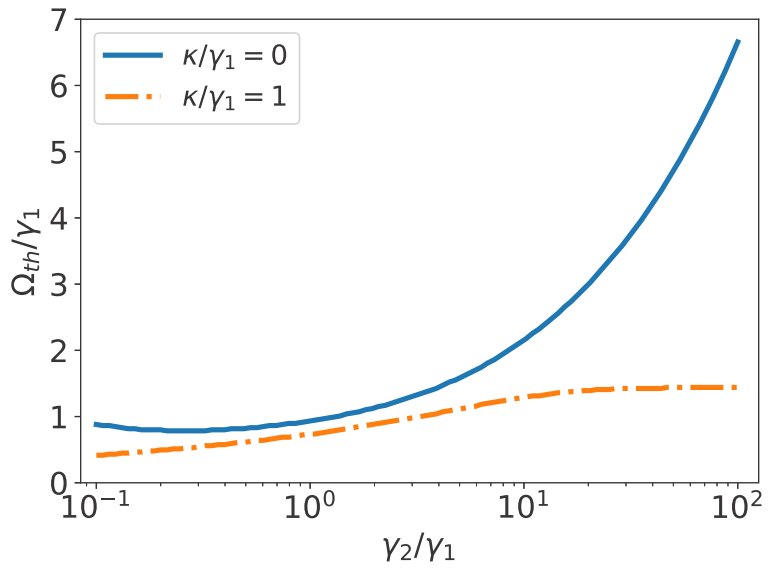

FIG. 5. Threshold driving strength $\Omega_{\text {th }} / \gamma_{1}$ against $\gamma_{2} / \gamma_{1}$, with the threshold set at $\epsilon=0.5$. Other parameters are: $\delta=\eta=0$. The qvdP limit cycle is more robust deeper in the quantum regime.

result is that single-photon dissipation cannot enhance synchronization if the damping ratio $\gamma_{2} / \gamma_{1}<3 / 4$ (corresponding to resonant driving), due to the larger decoherence for $\mathrm{vdP}$ limit cycles with larger amplitudes. The condition here is supported by numerical results in Fig. 3 which shows that no synchronization boost occurs when $\gamma_{2} / \gamma_{1}=1$. This shows that the dissipation boost is a purely quantum effect. The result here also aligns with our physical picture that the enhancement originates from the boost of the lowest coherence $\left|\rho_{01}\right|$, which has no classical analog.

\section{A. Robustness of the quantum vdP limit cycle}

Not only is synchronization boosted in the deep quantum regime, but the limit cycle is also robust to strong driving. We can invert Eq. (11) and find that in the deep quantum limit, $\epsilon$ is bounded from above by a constant, independent of driving strength:

$$
\begin{aligned}
\lim _{\Omega_{\mathrm{th}} / \gamma_{1} \rightarrow \infty} \epsilon & =\lim _{\Omega_{\mathrm{th}} / \gamma_{1} \rightarrow \infty} \frac{4 \Omega_{\mathrm{th}}^{2}\left(\kappa+\gamma_{1}\right)}{\gamma_{1}\left(4 \delta^{2}+\kappa^{2}+8 \Omega_{\mathrm{th}}^{2}+6 \kappa \gamma_{1}+9 \gamma_{1}^{2}\right)} \\
& =\frac{\kappa+\gamma_{1}}{2 \gamma_{1}}
\end{aligned}
$$

which reduces in the limit of $\kappa \rightarrow 0$ to $\epsilon=0.5$. This shows that quantum oscillators can be driven much harder than classical oscillators without breaking the assumption of selfsustained oscillations, further setting the qvdP apart from its classical counterpart. To demonstrate the robustness of the limit cycle, Fig. 5 shows numerical plots of $\Omega_{\text {th }} / \gamma_{1}$ against the "quantumness" $\gamma_{2} / \gamma_{1}$ using a threshold of $\epsilon=0.5$. Near the classical regime $\gamma_{2} / \gamma_{1}=0.1$, relatively weak driving is required to distort the limit cycle $\left(\Omega_{\mathrm{th}}<\gamma_{1}\right)$. In contrast, when the vdP is deeper in the quantum regime, stronger driving is required to achieve the same distortion, with $\Omega_{\text {th }} / \gamma_{1} \rightarrow \infty$ in the deep quantum limit. This shows the robustness of the vdP limit cycle, particularly in the deep quantum regime where $\Omega_{\text {th }} \gg \gamma_{1}$ is acceptable. However, Eq. (14) shows that the oscillator limit cycle becomes less robust with the addition of single-photon loss. An unlimited driving strength is also still not allowed, as it will break the assumptions of the Lindbladform master equation Eq. (1), namely that the driving can be added perturbatively without influencing the derivation of the dissipators [48].

\section{EFFECT OF SQUEEZING IS STRONGLY SUPPRESSED}

Here, we consider the standard $(\kappa=0)$ qvdP with a pure squeezing drive. Squeezing was reported to enhance frequency entrainment at the damping ratio $\gamma_{2} / \gamma_{1}=3$ [31]. It was claimed to open up the possibility of observing quantum synchronization in the deep quantum regime. The intuition is that frequency (and phase) locking in the quantum regime is inhibited by quantum fluctuations [10] which can be partially overcome by squeezing. The optimal combination of harmonic and squeeze driving has also been investigated [24]. However, we will now show that the advantage of squeezing is diminished as the ratio $\gamma_{2} / \gamma_{1}$ increases, and squeezing becomes ineffective for frequency entrainment in the deep quantum limit.

In Fig. 4, the observed frequency (in the rotating frame of the driving) $\delta_{\text {obs }}$, which is the spectral peak frequency, is plotted against driving strength and damping ratio $\gamma_{2} / \gamma_{1}$. In particular, Fig. 6(a) shows that when the harmonic driving strength increases, the amount of frequency shift is relatively insensitive to the damping ratio. In contrast, Fig. 6(b) shows that the entrainment due to squeezing decreases significantly
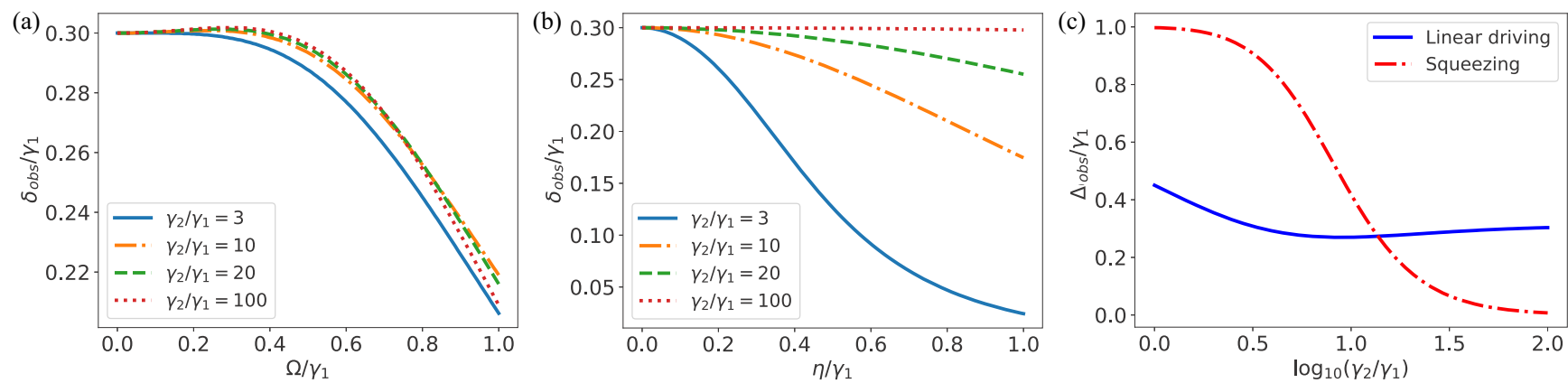

FIG. 6. (a) Observed frequency (in the rotating frame of the driving) against harmonic driving strength showing insensitivity of entrainment to $\gamma_{2} / \gamma_{1}$. (b) Observed frequency against squeezing strength showing the strong suppression of synchronization in the deep quantum regime. (c) Comparison between linear (or harmonic) driving and squeezing using relative entrainment (defined in main text). In all cases, $\delta=\kappa=0$. 
as $\gamma_{2} / \gamma_{1}$ increases, and becomes close to zero in the deep quantum regime. This difference in behavior can be observed in greater detail in Fig. 6(c), where the relative entrainment $\Delta_{\text {obs }}=\left(\delta_{\text {obs }}-\delta\right) / \delta$ is plotted against the damping ratio, for a fixed driving strength. For harmonic driving, the entrainment does not depend greatly on the damping ratio. However, squeeze driving results in strong entrainment (near unity) for $\gamma_{2}=\gamma_{1}$, but deteriorates to zero in the deep quantum limit. The crossover point where squeezing loses its advantage occurs at $\gamma_{2} / \gamma_{1} \approx 13$. We hereby emphasize that this is consistent with the result in Ref. [24] where the squeezing parameter $\eta$ has to increase with $\gamma_{2} / \gamma_{1}$ for a substantial synchronization effect. Our result thus complements that in Ref. [24] by showing that as compared to the case of harmonic driving (where synchronization is robust for all values of $\left.\gamma_{2} / \gamma_{1}\right)$, synchronization of a qvdP to a fixed squeezing strength is strongly suppressed in the deep quantum regime.

The level diagram in Fig. 4(c) explains why squeezing loses its effect in the deep quantum regime. Squeezing is a two-photon process, so it depends on the population of the state $|2\rangle$. In the deep quantum regime almost all the population is in the lowest two levels, so the squeezing drive decouples from the oscillator. This is another indication of new physics at $\gamma_{2} / \gamma_{1} \gtrsim 10$, motivating our definition as the threshold for the deep quantum regime.

\section{CONCLUSION}

In this paper we have investigated the quantum van der Pol oscillator in the deep quantum regime. The regime was identified as $\gamma_{2} / \gamma_{1} \gtrsim 10$ motivated by physical phenomena: We found that squeezing indeed provides a synchronization boost in the quantum regime, but in the deep quantum regime the effect is strongly suppressed compared to the robust synchronization from harmonic driving. Instead, we discovered a synchronization boost from single-photon dissipation, coming from a spontaneous decay of the oscillator into the environment. The effect is verified by exact numerical calculation of the master equation and analytical approximations. We also propose a physical explanation of the boost via the dynamics of the coherences in the density matrix, and show that such an effect with single-photon dissipation is not possible in the semiclassical and classical regimes of the qvdP. This synchronization boost is another example of noise-enhanced processes, this time unique to the quantum domain. Furthermore, our synchronization measure enables us to show that there is a maximal amount of synchronization in the deep quantum regime and that the oscillator can be driven much harder than its classical counterpart before the assumption of self-sustained oscillation breaks down. Our findings shed light on the fundamental physical differences between classical and quantum synchronization, and we believe that these results can act as fundamental building blocks for new phenomena in quantum nonlinear dynamics.

\section{ACKNOWLEDGMENTS}

The authors thank Tobias Haug for useful advice and the referees for their helpful feedback and suggestions which greatly improved the manuscript. The research is supported by the Ministry of Education and the National Research Foundation, Singapore.

\section{APPENDIX A: DENSITY MATRIX ELEMENTS}

The full expressions for the steady-state density matrix elements are given by

$$
\begin{gathered}
\rho_{00}=\frac{1}{D}\left(2 \gamma_{1}\left\{\gamma_{2}\left[4\left(\delta^{2}+\kappa^{2}\right)+6 \Omega^{2}\right]+3 \kappa\left(\kappa^{2}+2 \Omega^{2}\right)\right\}+\kappa\left(\gamma_{2}+\kappa\right)\left(4 \delta^{2}+\kappa^{2}+4 \Omega^{2}\right)+3 \gamma_{1}^{2} \kappa\left(7 \gamma_{2}+3 \kappa\right)+18 \gamma_{2} \gamma_{1}^{3}\right) \\
\rho_{11}=\frac{1}{D}\left\{\left(\gamma_{2}+\kappa\right)\left[\gamma_{1}\left(6 \gamma_{1} \kappa+9 \gamma_{1}^{2}+4 \delta^{2}+\kappa^{2}+12 \Omega^{2}\right)+4 \kappa \Omega^{2}\right]\right\} \\
\rho_{22}=\frac{1}{D}\left\{\gamma_{1}\left[\gamma_{1}\left(6 \gamma_{1} \kappa+9 \gamma_{1}^{2}+4 \delta^{2}+\kappa^{2}+12 \Omega^{2}\right)+4 \kappa \Omega^{2}\right]\right\} \\
\rho_{01}=\frac{1}{D}\left\{-2 \Omega\left[\gamma_{1}\left(\gamma_{2}-\kappa\right)+\kappa\left(\gamma_{2}+\kappa\right)\right]\left(-3 i \gamma_{1}+2 \delta-i \kappa\right)\right\}
\end{gathered}
$$

where the denominator $D$ is

$$
\begin{aligned}
D= & \gamma_{1}\left[4 \gamma_{1}\left(\delta^{2}+4 \kappa^{2}+3 \Omega^{2}\right)+15 \gamma_{1}^{2} \kappa+9 \gamma_{1}^{3}+4 \delta^{2} \kappa+7 \kappa\left(\kappa^{2}+4 \Omega^{2}\right)\right] \\
& +\gamma_{2}\left(3 \gamma_{1}+\kappa\right)\left(6 \gamma_{1} \kappa+9 \gamma_{1}^{2}+4 \delta^{2}+\kappa^{2}+8 \Omega^{2}\right)+\kappa^{2}\left(4 \delta^{2}+\kappa^{2}+8 \Omega^{2}\right)
\end{aligned}
$$

We can simplify the above expressions by taking the limit $\kappa \rightarrow 0$ (standard case). In this limit, the synchronization measure $S=\left|\rho_{01}\right|$ can be calculated to give

$$
\lim _{\kappa / \gamma_{1} \rightarrow 0} S=\frac{2 \gamma_{2} \Omega \sqrt{9 \gamma_{1}^{2}+4 \delta^{2}}}{4 \gamma_{1}\left(\delta^{2}+3 \Omega^{2}\right)+3 \gamma_{2}\left(9 \gamma_{1}^{2}+4 \delta^{2}+8 \Omega^{2}\right)+9 \gamma_{1}^{3}},
$$

which can be used to describe synchronization of the standard quantum van der Pol (qvdP) oscillator in the quantum regime (without going into the deep quantum regime). However, we can also consider the qvdP with single-photon loss in the deep 
quantum limit, for which the synchronization measure becomes

$$
\lim _{\gamma_{2} / \gamma_{1} \rightarrow \infty} S=\frac{2 \Omega\left(\gamma_{1}+\kappa\right) \sqrt{\left(3 \gamma_{1}+\kappa\right)^{2}+4 \delta^{2}}}{\left(3 \gamma_{1}+\kappa\right)\left(6 \gamma_{1} \kappa+9 \gamma_{1}^{2}+4 \delta^{2}+\kappa^{2}+8 \Omega^{2}\right)} .
$$

\section{APPENDIX B: SYNCHRONIZATION BOOST DUE TO SINGLE-PHOTON LOSS}

An intuitive picture to explain the synchronization boost is provided in the main text (in terms of the effects of singlephoton loss on the various coherences). One can also observe the boost by inspecting Eq. (8). In the deep quantum limit, since

$$
\left.\frac{\partial S}{\partial \kappa}\right|_{\kappa=0}=\frac{2 \Omega\left(3 \gamma_{1}^{2}+8 \Omega^{2}\right)}{\left(9 \gamma_{1}^{2}+8 \Omega^{2}\right)^{2}}>0
$$

for any fixed $\Omega>0$, there will still be an initial synchronization boost when increasing $\kappa$ from zero even after discounting the fact that $\Omega_{\text {th }}$ increases with $\kappa$. At the very least, we can conclude that synchronization in the deep quantum regime is highly robust against relaxation losses, a feature not present in the more classical case. This effect, of course, is not limited to only the deep quantum limit. Using the full expression of the density matrix, we can also impose the condition $\partial_{\kappa} S(\kappa=$ $0)>0$. To this end, we start with the synchronization measure

(a)

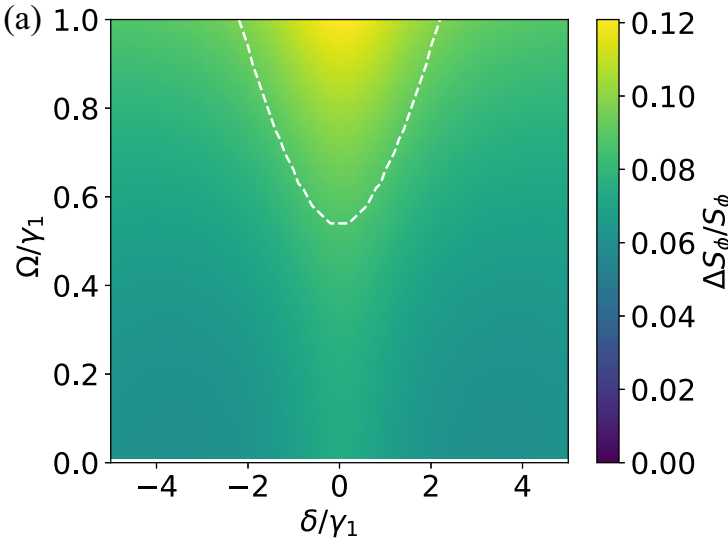

(without taking limits):

$$
S=\left|\rho_{01}\right|=\frac{M}{D},
$$

where

$$
N \equiv 2 \Omega\left[\gamma_{1}\left(\gamma_{2}-\kappa\right)+\kappa\left(\gamma_{2}+\kappa\right)\right] \sqrt{4 \delta^{2}+\left(\kappa+3 \gamma_{1}\right)^{2}}
$$

We assume $\gamma_{2}>\kappa$ such that $M>0$. The condition $\partial_{\kappa} S(\kappa=$ $0)>0$ is thus equivalent to [with $M^{\prime} \equiv \partial_{\kappa} M(\kappa=0)$ and similarly for $\left.D^{\prime}\right]$

$$
\frac{M^{\prime}}{M}>\frac{D^{\prime}}{D}
$$

Noting that both $D$ and $D^{\prime}$ are positive, a necessary condition for synchronization boost is therefore $M^{\prime} / M>0$. Substituting the expressions for $M^{\prime}$ and $M$ (evaluated at $\kappa=0$ ), the necessary condition is

$$
\frac{\gamma_{2}}{\gamma_{1}}>1-\frac{3}{4\left(\delta / \gamma_{1}\right)^{2}+12}
$$
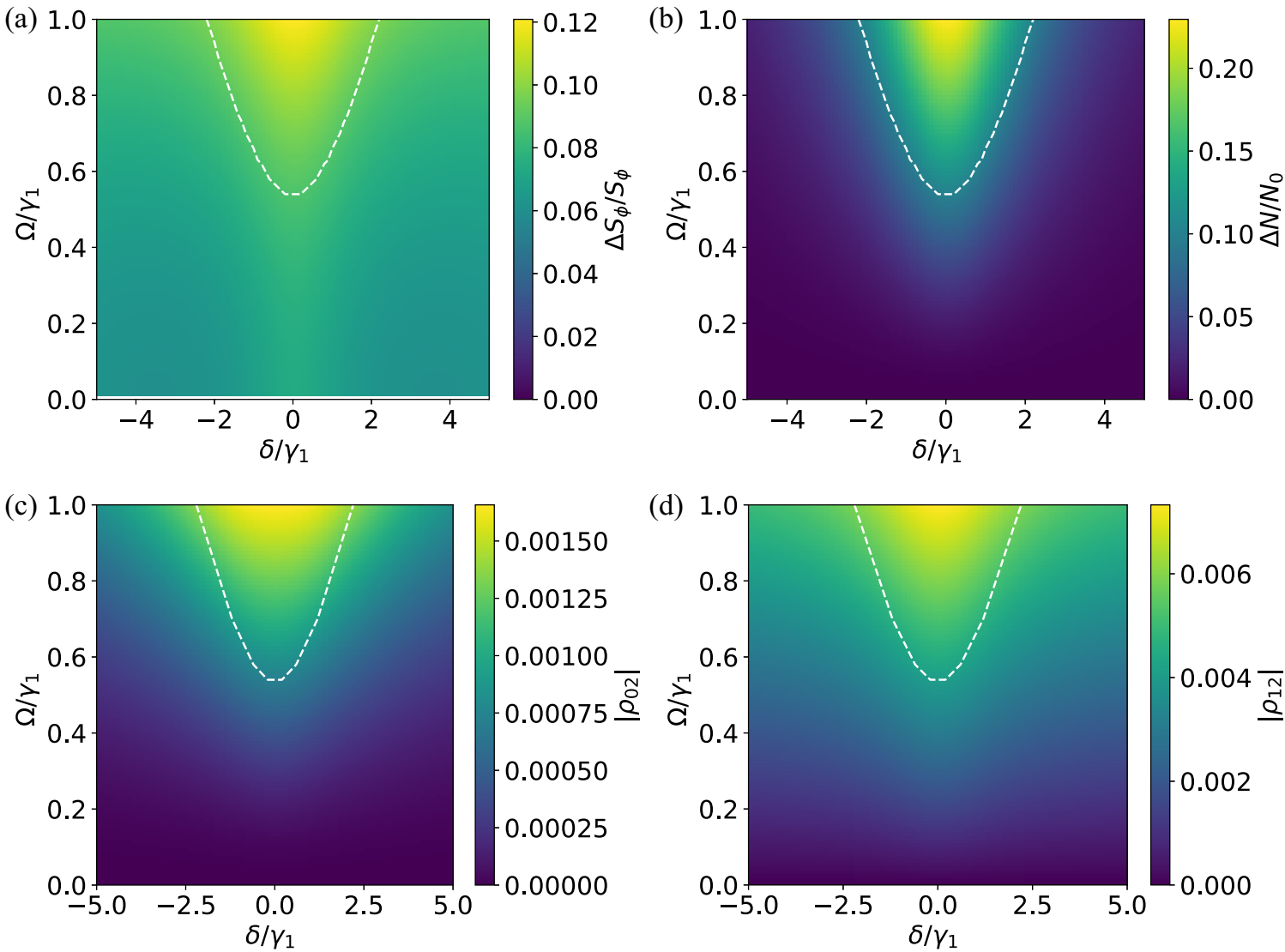

FIG. 7. (a) Difference of the Arnold tongue between numerical simulation and analytical solution. (b) Distortion of limit cycle, measured by the change in amplitude $\Delta N \equiv N-N_{0}$. (c) The magnitude of the coherence $\left|\rho_{02}\right|=|\langle 0|\rho| 2\rangle|$. (d) The magnitude of the coherence $\left|\rho_{12}\right|=$ $|\langle 1|\rho| 2\rangle|$. The white dashed line marks the threshold $\epsilon=0.1$. The damping ratio is set at $\gamma_{2} / \gamma_{1}=100$. 
In other words, if the damping ratio $\gamma_{2} / \gamma_{1}<3 / 4$ (corresponding to resonant driving), then it is impossible for single-photon loss to enhance synchronization. This shows that synchronization boost due to single-photon loss is a purely quantum effect. This also aligns with our physical picture that the enhancement originates from the boost of the lowest coherence $\left|\rho_{01}\right|$, which has no classical analog.

\section{APPENDIX C: COMPARISON OF THE ANALYTICAL AND NUMERICAL ARNOLD TONGUES}

Using Eq. (A6), the synchronization measure can be plotted against detuning and driving strength, giving the so-called Arnold tongue, which is a key signature of synchronization. The damping ratio $\gamma_{2} / \gamma_{1}$ is set as 100 to reach the deep quantum regime. The analytical solution agrees with the numerical simulation at low levels of driving. For stronger driving however, the analytical solution begins to differ from numerical results. This may be due to two reasons: (1) the coherence $\rho_{13}$ and/or $\rho_{23}$ becomes nonnegligible due to the driving, which thus breaks the assumption made in our solution, or (2) the external driving causes a significant distortion in the limit cycle, which contradicts the very definition of synchronization which requires the driving to only be a perturbation to the limit cycle.
To ensure that the limit cycle is not greatly distorted, one could set a threshold $\epsilon$ to impose the condition for synchronization that $\left|\Delta N / N_{0}\right|<\epsilon$, where $\Delta N \equiv N-N_{0}$. We set the maximum allowed distortion to be $\epsilon=0.1$. Note that while the choice of $\epsilon$ is arbitrary, $\epsilon$ has to be suitably small to prevent large distortions to the limit cycle. To quantify the accuracy of our analytical solution, we compute the difference between the synchronization measures, as shown in Fig. 7(a). Generally, using the threshold of $\epsilon=0.1$, the analytical solution is within around $10 \%$ accuracy of the the numerical simulation. The region with the most significant difference occurs where $\delta=0$ and relatively stronger driving, causing as high as $12 \%$ difference in the result. However, the region which exceeds the distortion threshold (marked out by the white dashed line) should not be regarded as synchronization due to the significant distortion of the limit cycle caused by driving.

For a better understanding of the slight inaccuracy of the analytical solution, we plot the magnitude of the coherences $\left|\rho_{02}\right|=|\langle 0|\rho| 2\rangle|$ and $\left|\rho_{12}\right|=|\langle 0|\rho| 2\rangle|$ in Figs. 7(c) and 7(d), respectively, where $\rho$ is the steady state density matrix. Unsurprisingly, both $\left|\rho_{02}\right|$ and $\left|\rho_{12}\right|$ are higher in the "disallowed" region exceeding the threshold $\epsilon=0.1$, primarily due to the stronger driving. It should also be noted however that $\left|\rho_{12}\right|>$ $\left|\rho_{02}\right|$. Again, this is not surprising because $\rho_{02}$ relies on two-photon process which is second-order in $\Omega$, thus explaining the small contribution when considering relatively weak driving.
[1] S. Strogatz, Sync: The Emerging Science of Spontaneous Order (Hyperion Press, New York, NY, 2003).

[2] A. Balanov, N. Janson, D. Postnov, and O. Sosnovtseva, Synchronization (Springer, Berlin/Heidelberg, 2009).

[3] A. Pikovsky, M. Rosenblum, and J. Kurths, Synchronization: A Universal Concept in Nonlinear Sciences, Vol. 12 (Cambridge University Press, Cambridge, UK, 2003).

[4] J. Cabral, E. Hugues, O. Sporns, and G. Deco, NeuroImage 57, 130 (2011).

[5] T. Kantermann, M. Juda, M. Merrow, and T. Roenneberg, Curr. Biol. 17, 1996 (2007)

[6] A. Chia, M. Hajdušek, R. Fazio, L.-C. Kwek, and V. Vedral, Quantum 3, 200 (2019).

[7] L. Gammaitoni, P. Hänggi, P. Jung, and F. Marchesoni, Rev. Mod. Phys. 70, 223 (1998)

[8] R. Benzi, A. Sutera, and A. Vulpiani, J. Phys. A: Math. Gen. 14, L453 (1981).

[9] T. E. Lee and H. R. Sadeghpour, Phys. Rev. Lett. 111, 234101 (2013).

[10] S. Walter, A. Nunnenkamp, and C. Bruder, Phys. Rev. Lett. 112, 094102 (2014).

[11] S. Walter, A. Nunnenkamp, and C. Bruder, Ann. Phys. 527, 131 (2015).

[12] N. Lörch, S. E. Nigg, A. Nunnenkamp, R. P. Tiwari, and C. Bruder, Phys. Rev. Lett. 118, 243602 (2017).

[13] N. Lörch, E. Amitai, A. Nunnenkamp, and C. Bruder, Phys. Rev. Lett. 117, 073601 (2016).

[14] L. Morgan and H. Hinrichsen, J. Stat. Mech.: Theory Exp. 09 (2015) P09009.
[15] A. Chia, M. Hajdušek, R. Fazio, L. Kwek, and V. Vedral, arXiv preprint arXiv:1711.07376 (2017).

[16] V. M. Bastidas, I. Omelchenko, A. Zakharova, E. Schöll, and T. Brandes, Phys. Rev. E 92, 062924 (2015).

[17] G. Heinrich, M. Ludwig, J. Qian, B. Kubala, and F. Marquardt, Phys. Rev. Lett. 107, 043603 (2011).

[18] M. Ludwig and F. Marquardt, Phys. Rev. Lett. 111, 073603 (2013).

[19] T. Weiss, A. Kronwald, and F. Marquardt, New J. Phys. 18, 013043 (2016)

[20] T. Weiss, S. Walter, and F. Marquardt, Phys. Rev. A 95, 041802(R) (2017).

[21] M. Bagheri, M. Poot, L. Fan, F. Marquardt, and H. X. Tang, Phys. Rev. Lett. 111, 213902 (2013).

[22] E. Amitai, N. Lörch, A. Nunnenkamp, S. Walter, and C. Bruder, Phys. Rev. A 95, 053858 (2017).

[23] A. Roulet and C. Bruder, Phys. Rev. Lett. 121, 053601 (2018).

[24] M. Koppenhöfer and A. Roulet, Phys. Rev. A 99, 043804 (2019).

[25] M. Koppenhöfer, C. Bruder, and A. Roulet, Phys. Rev. Research 2, 023026 (2020).

[26] J.-S. Zhang and A.-X. Chen, arXiv:1909.00536 (2019).

[27] Á. Parra-López and J. Bergli, Phys. Rev. A 101, 062104 (2020).

[28] J. Ilves, Bath-induced Persistent Rabi Oscillations in a Qubit, Master's thesis, Aalto University (2019).

[29] W. Wustmann and V. Shumeiko, Phys. Rev. Appl. 8, 024018 (2017). 
[30] D. Marković, J. D. Pillet, E. Flurin, N. Roch, and B. Huard, Phys. Rev. Appl. 12, 024034 (2019).

[31] S. Sonar, M. Hajdušek, M. Mukherjee, R. Fazio, V. Vedral, S. Vinjanampathy, and L.-C. Kwek, Phys. Rev. Lett. 120, 163601 (2018).

[32] A. W. Laskar, P. Adhikary, S. Mondal, P. Katiyar, S. Vinjanampathy, and S. Ghosh, Phys. Rev. Lett. 125, 013601 (2020).

[33] N. Jaseem, M. Hajdušek, V. Vedral, R. Fazio, L.-C. Kwek, and S. Vinjanampathy, Phys. Rev. E 101, 020201(R) (2020).

[34] A. Roulet and C. Bruder, Phys. Rev. Lett. 121, 063601 (2018).

[35] D. Witthaut, S. Wimberger, R. Burioni, and M. Timme, Nat. Commun. 8, 14829 (2017).

[36] V. Ameri, M. Eghbali-Arani, A. Mari, A. Farace, F. Kheirandish, V. Giovannetti, and R. Fazio, Phys. Rev. A 91, 012301 (2015).

[37] K. Ishibashi and R. Kanamoto, Phys. Rev. E 96, 052210 (2017).

[38] T. E. Lee, C.-K. Chan, and S. Wang, Phys. Rev. E 89, 022913 (2014).
[39] S. Dutta and N. R. Cooper, Phys. Rev. Lett. 123, 250401 (2019).

[40] N. Es' haqi Sani, G. Manzano, R. Zambrini, and R. Fazio, Phys. Rev. Research 2, 023101 (2020).

[41] Y. Kuramoto, Chemical Oscillations, Waves, and Turbulence, Vol. 19 (Springer Science \& Business Media, Berlin, 2012).

[42] K. V. Mardia and P. E. Jupp, Directional Statistics, Vol. 494 (John Wiley \& Sons, New York, 2009).

[43] C. Allefeld and J. Kurths, Int. J. Bifurcation Chaos 14, 405 (2004).

[44] D. T. Pegg and S. M. Barnett, Phys. Rev. A 39, 1665 (1989).

[45] H. Heimonen, A. Chia, M. Hajdušek, C. Noh, V. Vedral, and L. C. Kwek (unpublished).

[46] H. J. Carmichael, Statistical Methods in Quantum Optics 1: Master Equations and Fokker-Planck Equations (Theoretical and Mathematical Physics) (Springer, Berlin, 2003).

[47] Y. Kato, N. Yamamoto, and H. Nakao, Phys. Rev. Res. 1, 033012 (2019).

[48] G. Shavit, B. Horovitz, and M. Goldstein, Phys. Rev. B 100, 195436 (2019). 